\title{
marges Marges
}

revue d'art contemporain Revue d'art contemporain

$05 \mid 2007$

L'exposition sous toutes ses formes

\section{Art interactif et pré-cinéma. Des modes de relations parallèles et hybrides}

Interactive Art and Pre-cinema: Parallel and Hybrid Relational Modes

\section{Caroline Chik}

\section{(2) OpenEdition}

1 Journals

Édition électronique

URL : http://journals.openedition.org/marges/704

DOI : $10.4000 /$ marges.704

ISSN : 2416-8742

Éditeur

Presses universitaires de Vincennes

Édition imprimée

Date de publication : 15 juin 2007

Pagination : 22-39

ISBN : 978-2-84292-249-8

ISSN : $1767-7114$

\section{Référence électronique}

Caroline Chik, " Art interactif et pré-cinéma. Des modes de relations parallèles et hybrides », Marges

[En ligne], 05 | 2007, mis en ligne le 15 juin 2008, consulté le 10 décembre 2020. URL : http://

journals.openedition.org/marges/704; DOI : https://doi.org/10.4000/marges.704

Ce document a été généré automatiquement le 10 décembre 2020.

(c) Presses universitaires de Vincennes 


\title{
Art interactif et pré-cinéma. Des modes de relations parallèles et hybrides
}

\author{
Interactive Art and Pre-cinema: Parallel and Hybrid Relational Modes
}

\section{Caroline Chik}

1 L'œuvre interactive ne s'expose pas comme toute autre œuvre. La participation du spectateur, inhérente à la notion d'interactivité, crée de nouvelles relations œuvreespace-public ${ }^{1}$. Ainsi, le rapport à l'œuvre, et en particulier le rapport physique, s'en trouve changé. Il est des cas où le spectateur s'expose avec l'œuvre; il peut en outre se situer dans l'image, sur l'image ou encore faire image lui-même. Quant à l'œuvre, il ne suffit pas de la contempler : elle se manipule et se joue, à la manière d'un jouet. Nous verrons que la notion de contact par la main occupe une place importante dans la relation à l'œuvre interactive. Sous cet angle, nous mettrons cette dernière en parallèle avec les jouets et dispositifs optiques de l'époque pré-cinématographique. Certains modes de monstration et figures d'artiste hybrides de cette époque seront également rapprochés de ceux que l'on peut rencontrer dans l'art interactif. Ces parallèles s'inscrivent dans une réflexion sur l'héritage que constitue le pré-cinéma pour les artistes de l'interactivité.

\section{Le spectateur exposé}

2 Afin de pointer du doigt les mutations des modalités d'exposition induites par l'interactivité, nous proposons quelques constats sur la réception de l'œuvre interactive, en particulier sur ses à-côtés, ses aléas et ses paradoxes, pour nous pencher ensuite plus avant sur la relation étroite spectateur-image. 


\section{L'exigence de réciprocité}

3 On peut constater avec des installations interactives telles que Viens danser de Catherine Langlade que l'exposition de l'œuvre interactive implique parfois celle du spectateur luimême. Cette œuvre, au titre aussi enjoué qu'injonctif, propose ou plutôt demande au spectateur d'exécuter des pas de danse devant un écran installé en pleine ville, sous les yeux de passants perplexes et d'autres visiteurs en retrait, rivalisant d'immobilisme ${ }^{2}$. Le spectateur éprouve quelque réticence à jouer le jeu de la participation et à s'extraire des visiteurs discrets pour devenir acteur - ou « spectacteur ». L'art interactif met en jeu une relation cuvre-spectacteur-spectateurs, dont la dimension psychosociologique n'est pas négligeable. Forcé d'agir et de se donner en spectacle, le spectateur, s'il ne s'y plie pas, manque l'œuvre qu'il était venu "voir». La relation interactive semble reposer sur le paradoxe de la double contrainte (double bind), qui se résume à l'injonction «sois spontané ». Le spectateur est invité à agir de lui-même. Or sa participation n'est pas une éventualité, elle est de fait une nécessité. Principe essentiel à l'art interactif, elle constitue sa singularité, sa richesse, mais également son limite. L'existence de l'œuvre est fortement tributaire de la bonne volonté du spectateur qui, selon le principe de tout échange, est censé donner de sa personne pour recevoir l'œuvre. Ainsi, dans l'art interactif, la réception de l'œuvre ne va pas sans son corollaire: le don de la part du spectateur. Cette exigence de réciprocité, spécifique à l'art interactif, varie en intensité selon les œuvres qui développent, chacune, leur propre mode d'interaction.

\section{Le rôle rêvé}

Dans une démarche sensiblement différente, l'installation interactive On cherche la lumière de l'artiste chinois $\mathrm{Du}$ Zhenjun met judicieusement en lumière l'attirance irrésistible des mortels pour les feux des projecteurs, ainsi que leur instinct grégaire (voir ill. 1). L'artiste donne au spectateur le rôle "rêvé » en quelque sorte: celui d'un éclairagiste tout-puissant surplombant une fosse obscure. Lorsqu'il déplace un faisceau lumineux pointé vers le bas, en manipulant un joystick, un groupe d'individus en image vient s'agglutiner précisément sous la lumière, se bousculant parfois avec un autre groupe. Le spectateur se trouve dans une position dominante, quasi divine. La pénombre l'abritant des regards, il montre du doigt et se joue des bas instincts de l'homme. Mais peut-il oublier qu'il est homme lui-même ? Cette position, pour le moins idéale, dans laquelle le visiteur se place volontiers, Du Zhenjun semble la lui offrir avec une certaine ironie, l'interrogeant sur son comportement face à l'œuvre, face au groupe et finalement face au monde. 


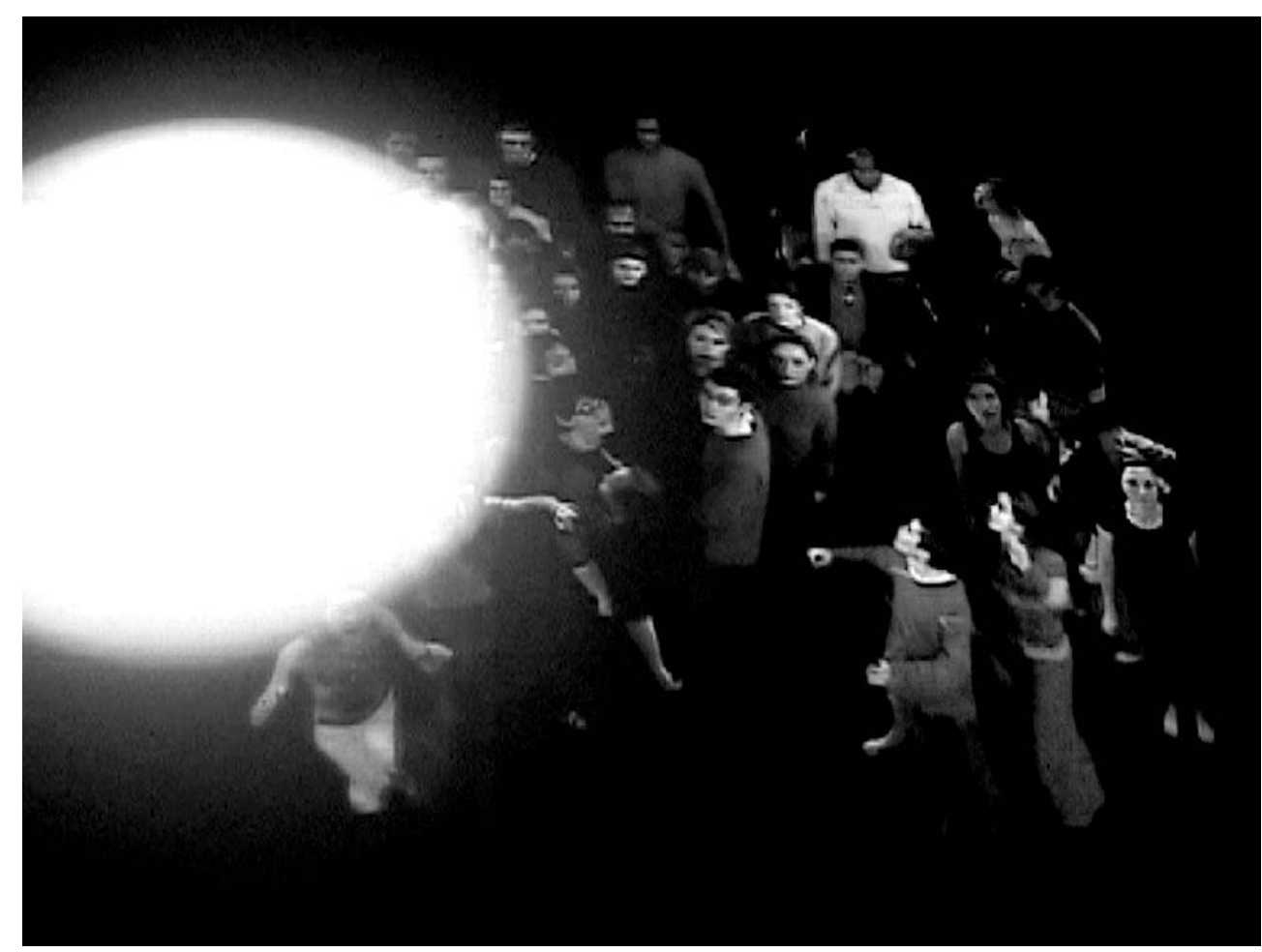

Installation interactive, « Cinémas du futur », Lille, 2003,

\section{Une question de distance}

Dans certains dispositifs interactifs à capteur de présence, il arrive que le spectateur distrait déclenche à son insu la projection d'une image sous ses pas. Mais n'ayant pas le recul physique nécessaire pour percevoir l'image par trop agrandie, il s'en éloigne parfois sans savoir qu'il marchait sur elle ${ }^{3}$. L'œuvre se comporte de manière paradoxale : invisible en l'absence de l'interacteur, elle peut l'être aussi lorsqu'il se joint à elle. Si au contraire il a conscience d'être intégré à l'œuvre, il lui faudra souvent imaginer ce qu'il est censé voir, n'identifiant au sol que de lumineuses étendues informes ou floues. Ce sont les spectateurs en retrait, situés à une certaine distance, qui profitent du spectacle de manière optimale. Dans ce cas, faudrait-il en conclure que l'installation interactive a besoin de «passants» (ou d'acteurs) pour exister et de spectateurs (passifs) pour être appréciée?

\section{Le spectateur mis en image}

6 Outre le corps même du spectateur, il n'est pas rare de voir son image intégrée à l'œuvre. Caméra vidéo et écran étant souvent collés l'un à l'autre, le spectateur filmé se voit en train d'être vu en train de (se) regarder... L'écran se fait miroir, le spectateur se fait image. En 1984, l'artiste vidéaste Bill Viola voyait en l'avènement de l'informatique dans l'art «la fin de la caméra», rappelle Florence de Mèredieu dans Arts et nouvelles technologies ${ }^{4}$. Or aujourd'hui, la caméra vidéo numérique, qui conjugue saisie optique du réel et propriétés numériques, constitue un outil indispensable pour nombre d'artistes de 
l'interactivité, que ce soit dans la création de l'œuvre ou au moment de sa réception - ne serait-ce qu'en tant que capteur de présence. L'incorporation de l'image du visiteur captée par une caméra est le principe de Fantômes ${ }^{5}$ (2005), installation interactive de Vincent Lévy: l'image des spectateurs se fond avec celle d'autres visiteurs filmés antérieurement au même endroit. Deux couches temporelles se superposent dans la même image : le présent du direct (spécifique à l'image vidéo) et le passé de l'image enregistrée. Tous deux sont mis à plat par le temps de l'ordinateur, appelé le temps réel. Le faux miroir qu'est l'écran fait apparaître des résurgences de spectateurs; on réalise alors que l'illusion existe aussi dans l'art interactif : elle porte sur la rencontre paradoxale et l'ambiguïté entre présence passée et présente.

\section{Comme l'image, le spectateur}

7 Non seulement le spectateur fait partie de l'image interactive, s'expose en elle mais par son comportement, il lui ressemble parfois. Une une sorte de mimétisme spectateurimage. Ainsi dans une animation vidéo interactive l'image alterne généralement action et attente d'action. De même, face à elle, le spectateur va osciller entre agir et s'immobiliser, que ce soit dans l'attente du «bon » moment avant de cliquer, ou bien pour comprendre ce qui se passe lorsqu'il n'agit pas. C'est dire que dans la relation interactive, les arrêts et les non-actions sont aussi décisifs que leurs contraires. Agir / ne pas agir: ce double comportement ne reflète-t-il pas le schème binaire inhérent à l'informatique - alternance du zéro et du un ? Cette binarité semble déteindre sur l'œuvre elle-même, au niveau de sa structure programmatique, comme au niveau du choix d'actions possibles pour le spectateur (cliquer / relâcher, tourner une mollette à gauche / à droite), ou encore de l'image même, qui souvent se dédouble en diptyque ou sur deux écrans différents. Liée à l'origine à une contrainte technique, cette binarité s'avère féconde, présente de manière obsessive dans certaines œuvres ${ }^{6}$.

8 Le comportement du spectateur peut concerner soit le corps entier - œuvres immersives -, soit la main seule - œuvres vouées à la configuration domestique écran-souris (cd ou dvd-rom, Net art). Arrêtons-nous sur ce comportement manuel du spectateur.

\section{Regarder l'œuvre avec les mains : une esthétique du contact}

$9 \quad$ L'interdiction de toucher n'est pas de rigueur dans l'art interactif (au contraire de l'art traditionnel). Plus exactement, l'interactivité incite moins au toucher qu'au contact. L'intérêt, selon nous, ne portant pas tant sur le toucher que sur ce qui se produit. Le contact fait acte, puisque le spectateur va actualiser l'image en latence, en attente. Le trouble du contact interactif naît dans l'alliance du plaisir de voir et de faire, simultanément ou dans des temps rapprochés.

Il est question de regarder l'image et d'agir sur elle, par le biais de la souris ou de toute autre interface censée être maniée. On peut voir dans le fil de la souris la matérialisation d'un « cordon ombilical ${ }^{7}$ » retrouvé qui lierait le spectateur à l'image. Quand bien même la souris ne possèderait plus de fil, il reste la souris; si le contact s'établit sans souris, alors reste la main, la main comme interface. Dans tous les cas, la dématérialisation des relations tant commentée n'est pas totale. 
11 La main du spectateur - qu'il faudrait nommer ici manipulateur - prend une place essentielle dans l'art interactif et de manière si évidente qu'on y prête rarement attention. Or, elle n'a certainement pas le même statut que celle du lecteur de livre imprimé. Jean-Louis Boissier remarque : «Il faut [aussi] s'interroger sur le retour de la main, comme Roland Barthes l'a fait à propos des planches de l'Encyclopédie ${ }^{8} »$.

12 L'art interactif, du point de vue de l'histoire de l'art et des media, n'est pas seulement le fruit d'une révolution numérique, mais en amont de celle-ci, le produit du passage d'un art manuel à un art technologique né au $19^{\mathrm{e}}$ siècle. L'immatérialité engendrée par les technologies numériques nous ferait presque oublier l'origine manuelle des outils. Aujourd'hui la main devient moins essentielle à l'artiste. De surcroît, à mesure qu'elle perd de son importance dans la création, il semble qu'elle en gagne sur le plan de la réception, dans la relation œuvre-spectateur et / ou dans le sujet-même de l'œuvre.

En effet, la main occupe une place centrale dans nombre d'œuvres récentes. L'image, projetée sur une table-écran, se trouve à portée de main du spectateur. Dans l'installation Se toucher toi de Grégory Chatonsky (voir ill. 2), le spectateur déplace sa main sur un écran horizontal afin de provoquer - ou non - le contact entre une main féminine et une main masculine, dans un jeu binaire de rapprochement / éloignement. Michaël Cros, avec Les Mains (voir ill. 3), propose au visiteur d'entrer en contact et d'agir manuellement sur le comportement et le destin de mains, entités séparées de tout corps. Cette œuvre, ainsi que quelques autres précitées, fut présentée au bien nommé festival « $1^{\mathrm{er}}$ contact ».

\section{2 Gregory Chatonsky, Se toucher toi}

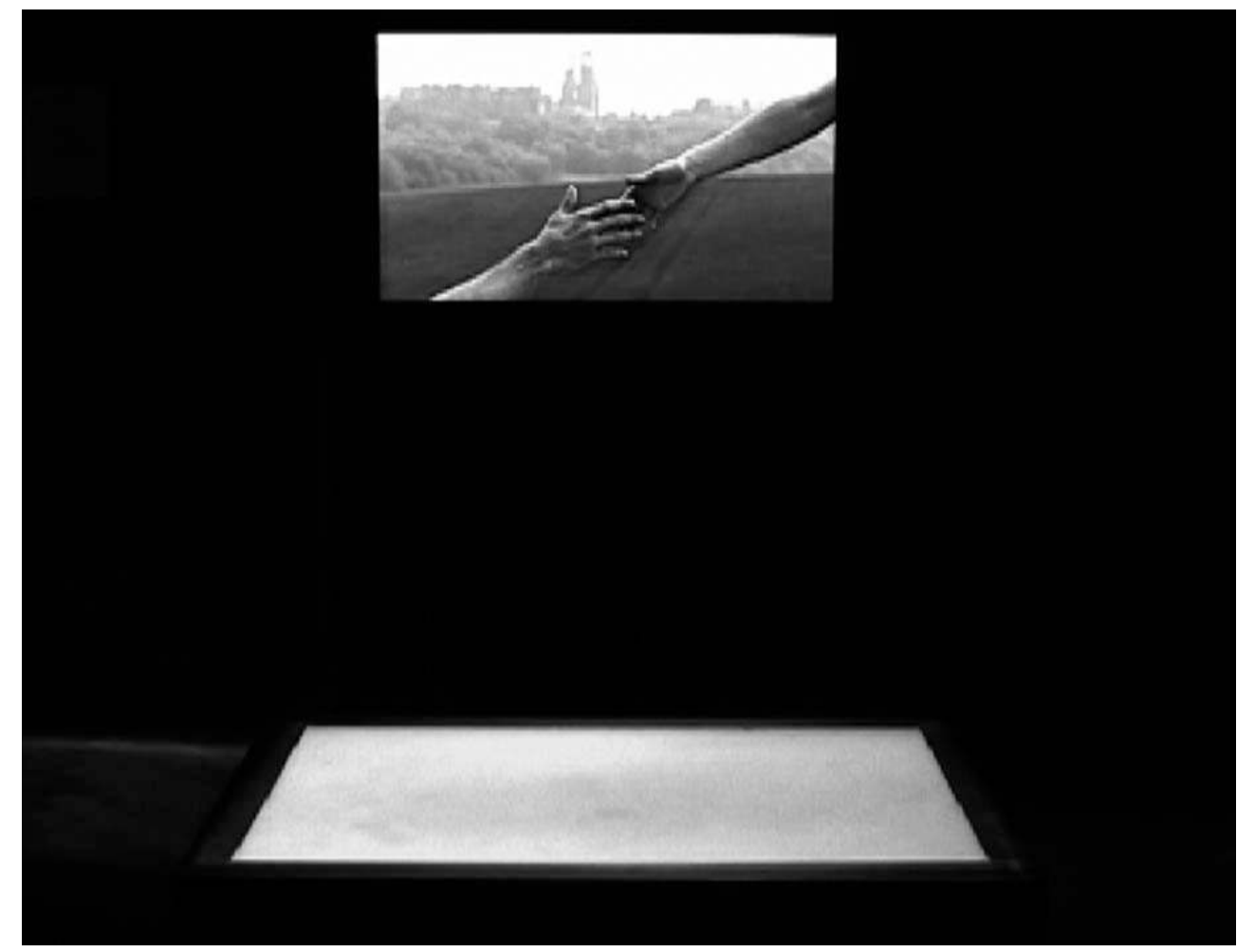

Installation interactive, «Transmissions », Maison internationale de la cité universitaire, Paris, 2005. Version internet : http://www.incident.net/works/touch/ 


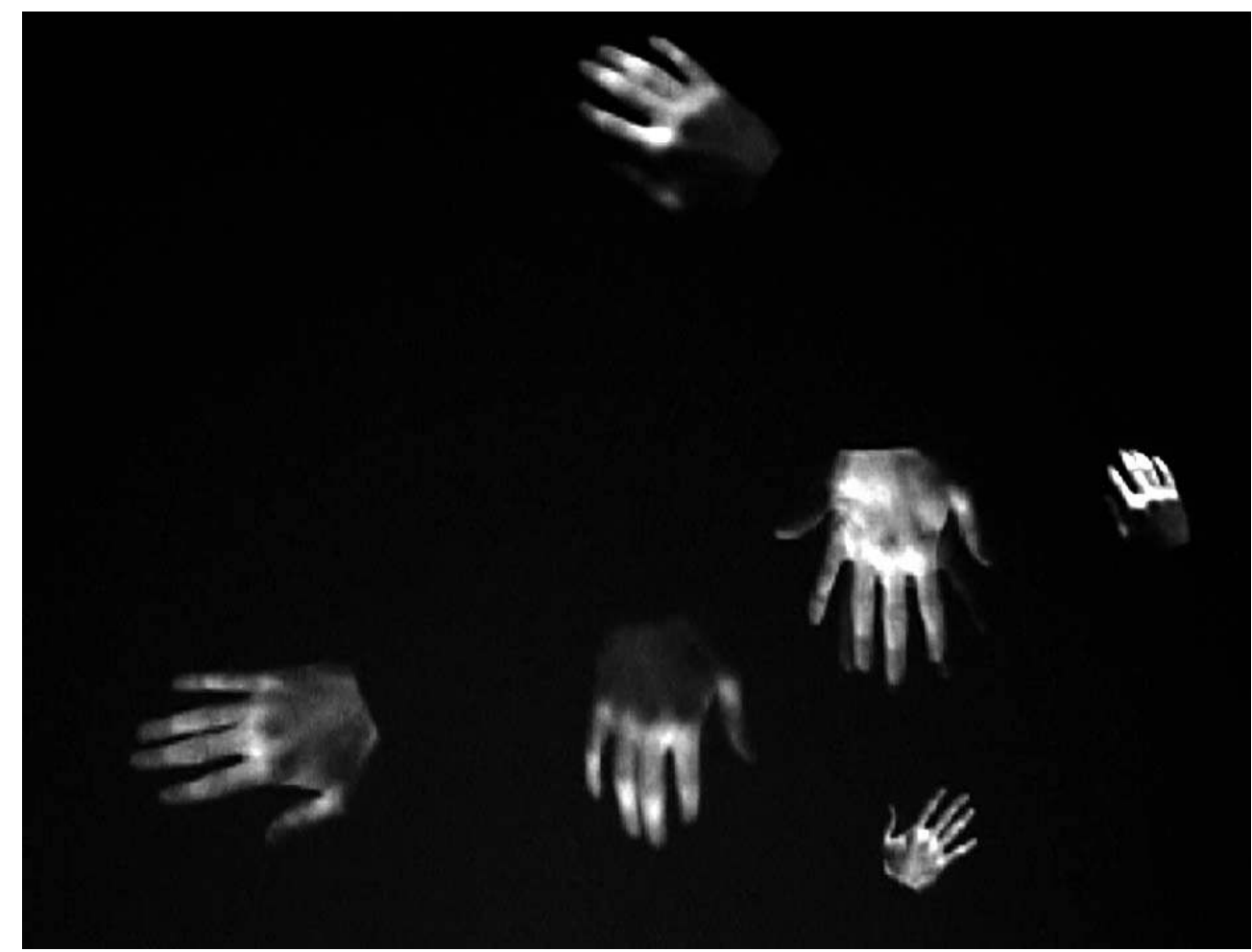

Installation comportementale interactive, « $7^{\mathrm{er}}$ contact», Issy-les-Moulineaux, 2004.

14 La main comme interface invente l'écran horizontal, appelé « table sensitive » par JeanLouis Boissier, « table haptique » ou encore « hypertable » par Douglas Edric Stanley. L'un et l'autre développent cette interface dans différentes installations interactives. Dans $L a$ Morale sensitive (2001-2003) ou Le Petit Manuel interactif (2001) Jean-Louis Boissier assimile l'horizontalité de l'image ou du texte à celle du livre; la table et la chaise d'écolier tendent à renforcer ce rapprochement. La grande table-écran de Concrescence de Douglas Stanley réfère quant à elle davantage à la toile du cinéma (voir ill. 4). Ainsi le film interactif se regarde avec les mains. 
III. 4 Douglas Edric Stanley, Concrescence

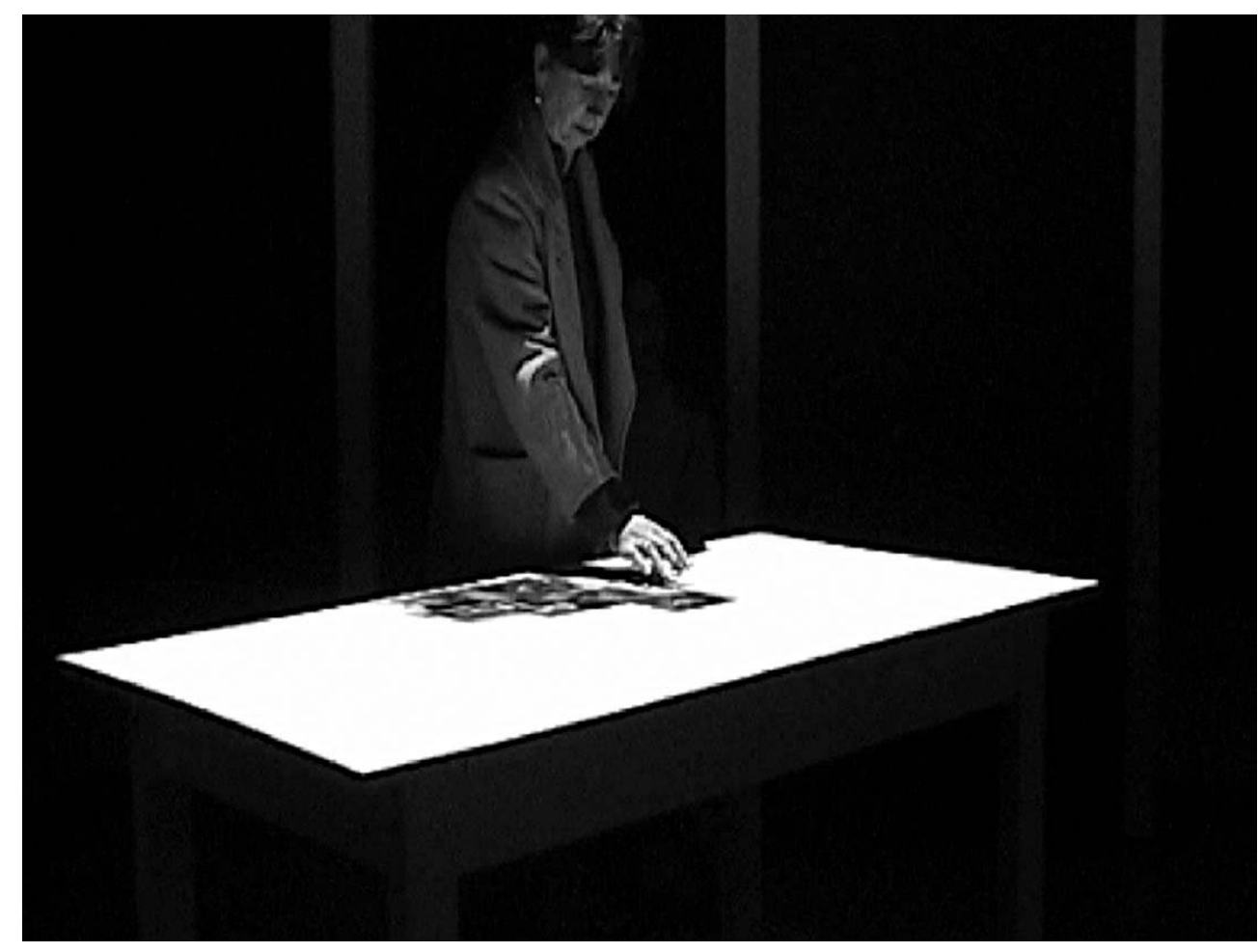

Dispositif de cinéma algorithmique, « Nemo », Forum des images, Paris, 2003.

15 Eric Lanz s'intéresse à la question de la main dans son rapport à l'outil. De ce prolongement de la main qu'est l'outil, découlent les technologies les plus avancées. À travers ses différentes œuvres vidéo et interactives (dont Manuskript ${ }^{9}$ ), Eric Lanz espère «rappeler au spectateur qu'il est en rapport avec des images, qui ne sont pas immédiates ». Dès 1993 il considère que « l'évolution technologique à laquelle on assiste [...] a tendance à effacer, à faire oublier cette médiatisation : on a l'impression de pouvoir tout faire, tout créer, tout atteindre dans un monde virtuel. Alors qu'on est peut-être en train de s'éloigner des choses, d'avoir moins d'influence sur elles ${ }^{10} »$. Son travail autour de la main cristallise sa réaction face à ce constat.

16 L'image interactive, bien que bidimensionnelle, distante ou immatérielle, se comporte en image-objet. Mobile, maniable, tangible, manipulable ou encore manœuvrable, nous allons voir qu'elle n'est pas sans rapport avec le modèle du jouet.

\section{Euvre-jouet et dispositifs pré-cinématographiques}

17 Plus d'un siècle avant l'apparition de l'interactivité existait une autre figure de spectateur hybride usant de ses mains autant que de ses yeux. Ce que l'on nomme le pré-cinéma, dont la période la plus féconde est le $19^{\mathrm{e}}$ siècle - celui de la révolution industrielle et des prémisses de la technologie de masse -, a vu apparaître un large éventail de dispositifs, de machines à voir et à animer, de jouets d'optique très prisés, luxueux ou modestes. Les phénakistiscopes, zootropes ou autres praxinoscopes (voir ill. 5 et 6) sont des jouets qui exploitent l'impression de mouvement à partir d'images fixes (dessins, gravures, puis 
chronophotographies) ${ }^{11}$. Ces objets, aujourd'hui archaïques, étaient alors à l'avant-garde de la technologie, comme le sont les jouets et installations interactifs.

L'électricité n'avait pas encore fait son entrée dans la sociétée ${ }^{12}$. On utilisait encore la main, la manivelle qu'on actionnait pour le plaisir des yeux. Le pré-cinéma avait donc introduit l'idée d'un cinéma qui se joue et se manipule.

III. 5 Zootrope, avec ses bandes

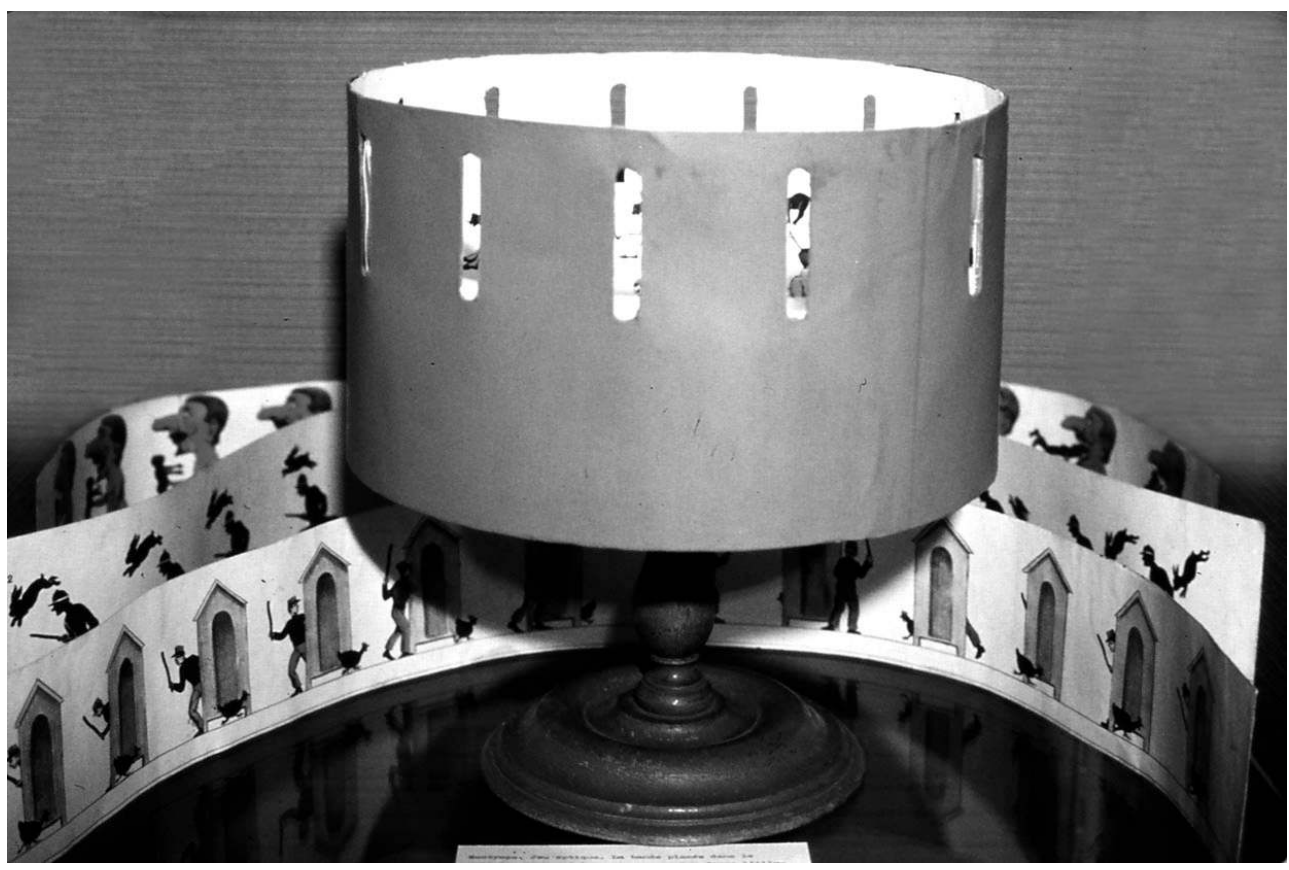

Début du $20^{e}$ siècle. Carton, papier et bois, collection du Musée du Jouet, Poissy 


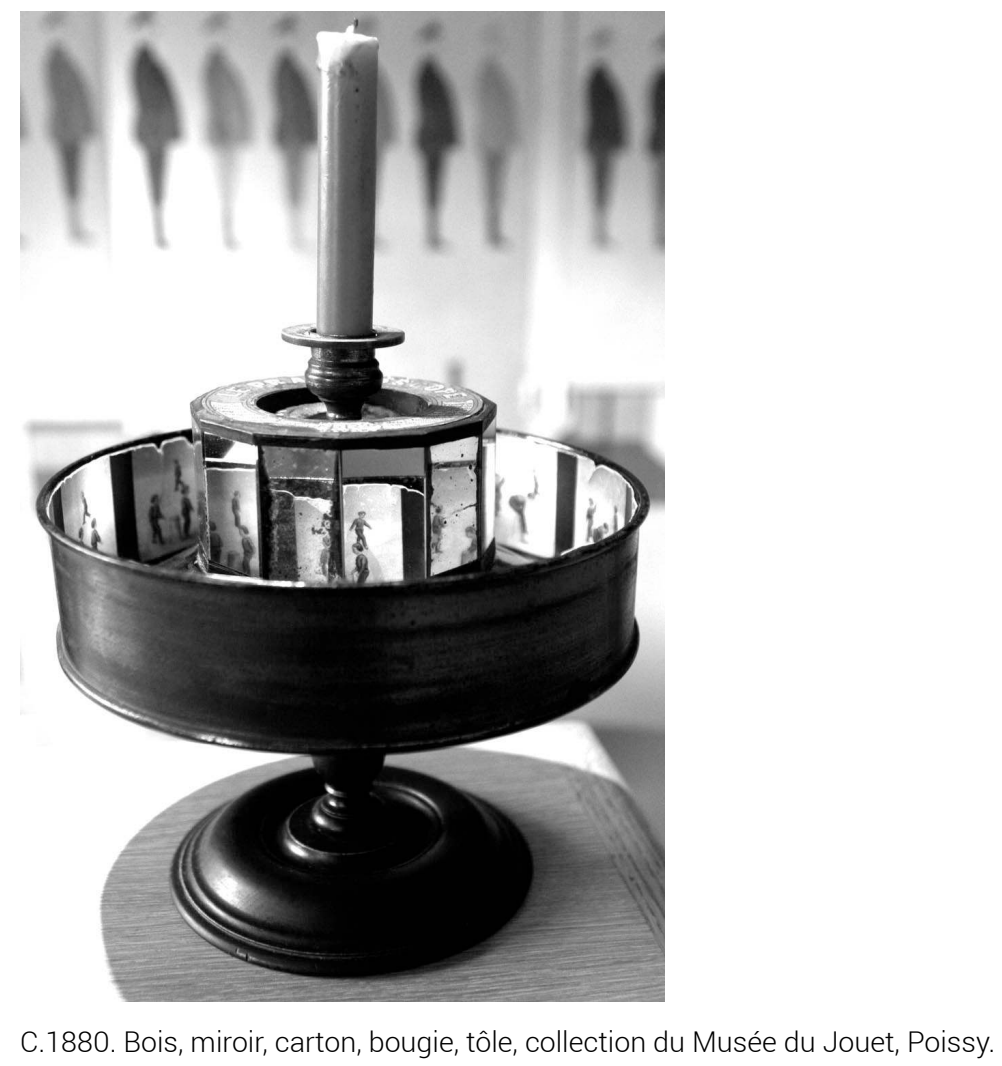

\section{Une pré-interactivité ?}

19 Nous ne sommes pas en train d'avancer que le pré-cinéma était déjà interactif. L'interactivité est un terme essentiellement informatique apparu au $20^{\mathrm{e}}$ siècle. Toutefois nous voulons attirer l'attention - à travers les notions de jouet, de spectacle, de contact manuel, de comportement des utilisateurs - sur les liens étroits entre les spectateurs et les œuvres hybrides de deux époques si éloignées. En ce sens, de même que l'on parle a posteriori de pré-cinéma, peut-être pourrait-on aussi bien repenser cette époque en terme de pré-interactivité, la filiation fût-elle moins directe, notamment d'un point de vue technique.

On situe communément les origines de l'art interactif ou "participatif » dans l'art cinétique, ou encore l'art conceptuel. Or de nombreux travaux artistiques (ceux des jeunes générations surtout) s'inscrivent en marge de cette filiation et semblent se situer moins dans le sillage de "l'art à l'ordinateur » que dans celui du cinéma. Ces artistes s'interrogent sur les suites possibles du septième art en redécouvrant ses origines et ses diverses formes, prenant conscience qu'il est aussi une technologie, un spectacle, une installation et un dispositif spatio-temporel. Ils déconstruisent les codes du cinéma classique, réexplorent le cinéma expérimental, le cinéma des premiers temps, ou encore l'art « optico-manuel » qu'est le pré-cinéma.

21 Art non noble, le pré-cinéma est pourtant « le grand art de la lumière et de l'ombre ${ }^{13}$ ", comme l'écrit Laurent Mannoni, spécialiste de cette "haute époque» du cinéma. Mannoni s'attache à faire sortir de l'ombre cet art de la lumière, et tente d'en retracer 
l'histoire sans la réduire à son seul aspect technique. Selon Dominique Païni, il se penche davantage «sur des zones parallèles, plus obscures, parfois secondaires de l'histoire de l'art, que cette dernière a repoussées avec force dans ses marges. $Y$ aurait-il, après tout, deux histoires de l'art ${ }^{14}$ ? ». La référence, voire l'hommage rendu par des artistes actuels aux jouets et dispositifs pré-cinématographiques permettra peut-être la légitimation artistique de ce pré-cinéma.

\section{Un « archaïsme de pointe »}

Aujourd'hui, on retrouve chez le spectateur interacteur des comportements similaires à ceux $d u$ "manipulecteur» ou encore du projectionniste du $19^{e}$ siècle. Un nombre croissant d'artistes de l'interactivité s'intéressent à une certaine " archéologie des media » et surtout à ces jouets et dispositifs du pré-cinéma. En témoignent certaines œuvres que l'on a pu voir dans la grande exposition «Future Cinema » au zkm (Karlsruhe) - dont le catalogue conséquent fait figure de manifeste ${ }^{15}-$, ou dans « Cinémas du futur » à Lille et à Créteil, qui ont permis de faire découvrir à un large public les liens profonds entre art interactif et pré-cinéma.

Le Japonais Toshio Iwai, auteur de phénakistiscopes modernes (voir ill. 7), travaille sur cette question depuis longtemps: "Enfant je passais beaucoup de temps à écrire des textes de bandes dessinées sur mes livres de cours et à créer des jouets à moteur. À l'époque, ils représentaient pour moi la pointe de la technologie. J'ai l'impression que c'est à ce moment que j'ai commencé à créer et j'ai continué ensuite sous l'impulsion de l'évolution technologique. Il me semble que le fait d'avoir eu entre les mains des outils comme les vidéos, les ordinateurs et Internet et d'avoir pris plaisir à les utiliser a fait naître beaucoup de mes œuvres. J'ai recherché le sentiment de mon enfance dans l'univers du numérique et c'est la raison pour laquelle j'ai continué à exprimer les rapports entre les media, la machine et les gens à travers des œuvres interactives ${ }^{16}{ }^{\prime}$. Qu'il se manifeste sous la forme du jouet ou bien qu'il symbolise l'enfance de l'art "technologique », on peut observer que l'univers de l'enfance y est omniprésent. Les œuvres interactives et le site internet de l'artiste Zoe Beloff ${ }^{17}$ offrent un voyage temporel dans l'univers magico-ludique du pré-cinéma. La page internet Illusions (Philosophical Toy World) propose d'expérimenter différents jouets optiques. La transposition interactive de ces jouets (tels que le thaumatrope) n'est pas difficile : ils trouvent tout naturellement un second souffle dans le jouet interactif qu'est l'ordinateur. L'installation de Tomohiro Sato Floating Memories ${ }^{18}$ (2002) met en place une boîte à musique interactive qui invite le spectateur à produire non seulement du son mais aussi des images : une caméra filme le visage du visiteur, lequel, en actionnant la manivelle, projette son image, sous la forme d'une succession de photogrammes, projetés sur une bande de papier vierge. La manipulation manuelle, typique de l'ère mécanique, contraste avec les moyens techniques numériques mis en œuvre dans ce dispositif. 
III. 7 Lanterne magique avec plaques de verre

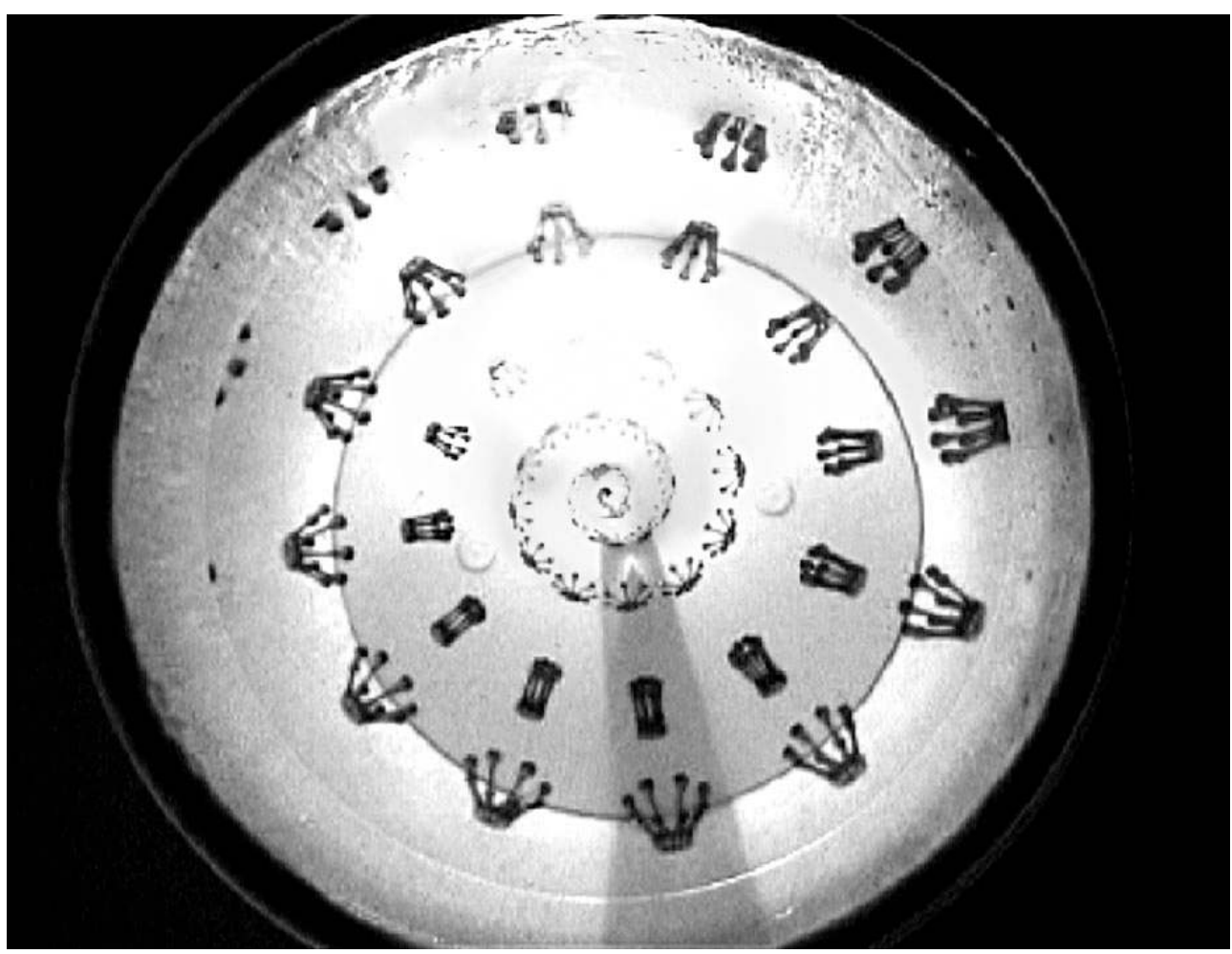

Début du $20^{\circledR}$ siècle. Fer blanc, verre et décalcomanie, collection du Musée du Jouet, Poissy.

\section{Le spectateur-joueur, l'œuvre-jouet}

Si le spectateur est semblable à un joueur, il nous paraît intéressant de voir en lui non pas le rapport au jeu mais au jouet. Le joueur de l'art interactif serait peut-être à considérer moins en tant que gamer, joueur de jeux vidéo, auquel on n'a de cesse de le comparer, qu'en tant que manipulateur d'œuvre-jouet.

Le jouet n'est pas seulement le jouet d'enfant, il est aussi « jouet philosophique » - ainsi que Zoe Beloff nomme les jouets d'optique qu'elle réactualise. Le jouet procure une sensation instantanée de plaisir intellectuel et esthétique ${ }^{19}$, dans le moment même où il est manipulé. Ce qui n'est pas sans conséquences sur la temporalité de l'œuvre et de sa réception par le spectateur-joueur. Celui-ci s'attache à l'acte en train de se faire et non plus seulement à ce qu'il va provoquer. Il apprécie la conséquence de ses actes en même temps qu'il les génère. Il ne tend pas nécessairement vers un but final, vers un temps à venir comme le gamer du jeu vidéo. Dans les œuvres-jouets que nous envisageons, tout converge vers le présent du spectateur. L'immédiateté permise par le temps réel est mise en avant. Les œuvres interactives fondées sur le long déroulement d'un parcours semé d'obstacles et censé mener à la victoire placent souvent le pseudo gamer dans une posture d'attente de quelque chose qui ne viendra généralement pas : une issue, une victoire, la vague sensation d'avoir gagné quelque chose. On ne peut pas jouer le double jeu théorique de comparer le spectateur à un gamer et constater avec impuissance la quantité d'œuvres interactives déceptives. 
On objectera peut-être que le jouet revêt "moins d'interactivité » que le jeu, plus complexe. Mais il est évident que l'interactivité dans l'art n'est pas une question de degré hypothétique. L'interactivité nous paraît intéressante pour le contact, la relation à l'homme qu'elle crée, et non pour la technicité qu'elle représente. Appréhender l'œuvre interactive comme un jouet plutôt qu'un jeu vidéo la libère de la contrainte du récit, de la tension vers un but, de la linéarité et finalement de modèles logiques ou narratifs qui ne lui conviennent pas forcément. Le jouet implique l'immédiateté et la matérialité, éléments à prendre en compte en vue de développer de nouveaux modes de relation à l'œuvre.

\section{Résonance entre pré-cinéma et arts interactifs}

\section{Le livre-jouet}

Le jouet d'optique possède des traits communs avec l'œuvre interactive. Spectacle miniature et individuel tenant dans la main, il est la plupart du temps un objet que l'on peut acquérir. C'est le cas du livre-jouet, qui a pris diverses formes et appellations telles que livre magique, animé, à mécanisme, à système ou à transformation, jusqu'à son avatar actuel sous le nom de pop up. Il ne dévoile sa magie qu'une fois saisi et animé par le lecteur-joueur. Florence de Mèredieu, mais aussi Jean-Louis Boissier, ont établi cette analogie entre œuvres sur cd-rom et livres animés, qui sont les « ancêtres de nos tableaux interactifs, puisque images animées, à animer, incluses dans un volume ${ }^{20}$ ».

Le flip book ou folioscope (d'abord appelé flick-book et kineograph) est une forme très cinématographique du livre-jouet. Le feuilletage rapide des pages entre le pouce et l'index provoque l'impression de mouvement des images, et transforme le livre minuscule en un « cinéma de poche ${ }^{21}$ ». La main active du spectateur-feuilleteur est une fois encore la condition de possibilité d'animation de l'image.

\section{La lanterne magique, spectacle et jouet}

La lanterne magique ${ }^{22}$ (voir ill. 8) représente une figure particulière et protéiforme de monstration des images. Privée ou publique, familiale, savante ou foraine, la séance de projection à la lanterne, spectacle apparaissant dès le $17^{\mathrm{e}}$ siècle, combine images, sons, paroles et écrits. Cette hybridation « n'est pas sans rapport avec l'histoire du livre illustré et de la bande dessinée, du cinéma et du dessin animé, et enfin, de l'audiovisuel et du multimédia, ces langages visuels du $20^{e}$ siècle ${ }^{23}$ ", comme le remarque Ségolène le Men. À l'ère de l'industrialisation, l'appareil se miniaturise : la lanterne jouet va supplanter peu à peu la lanterne de projection. Signe de l'évolution technologique, la miniaturisation se poursuit aujourd'hui. Le cd ou le dvd-rom artistique, à l'instar de la lanterne, non seulement sont des objets multimédia types, mais possèdent la même double vocation: objets commercialisés destinés à un usage ou une "projection » privés, ils s'exposent également sous forme d'installation-projection publique. En outre le cd-rom peut constituer, au même titre que la lanterne magique, un outil scientifique ou ludo-éducatif. 
III. 8 Toshio Iwai, Phenakistiscope of light

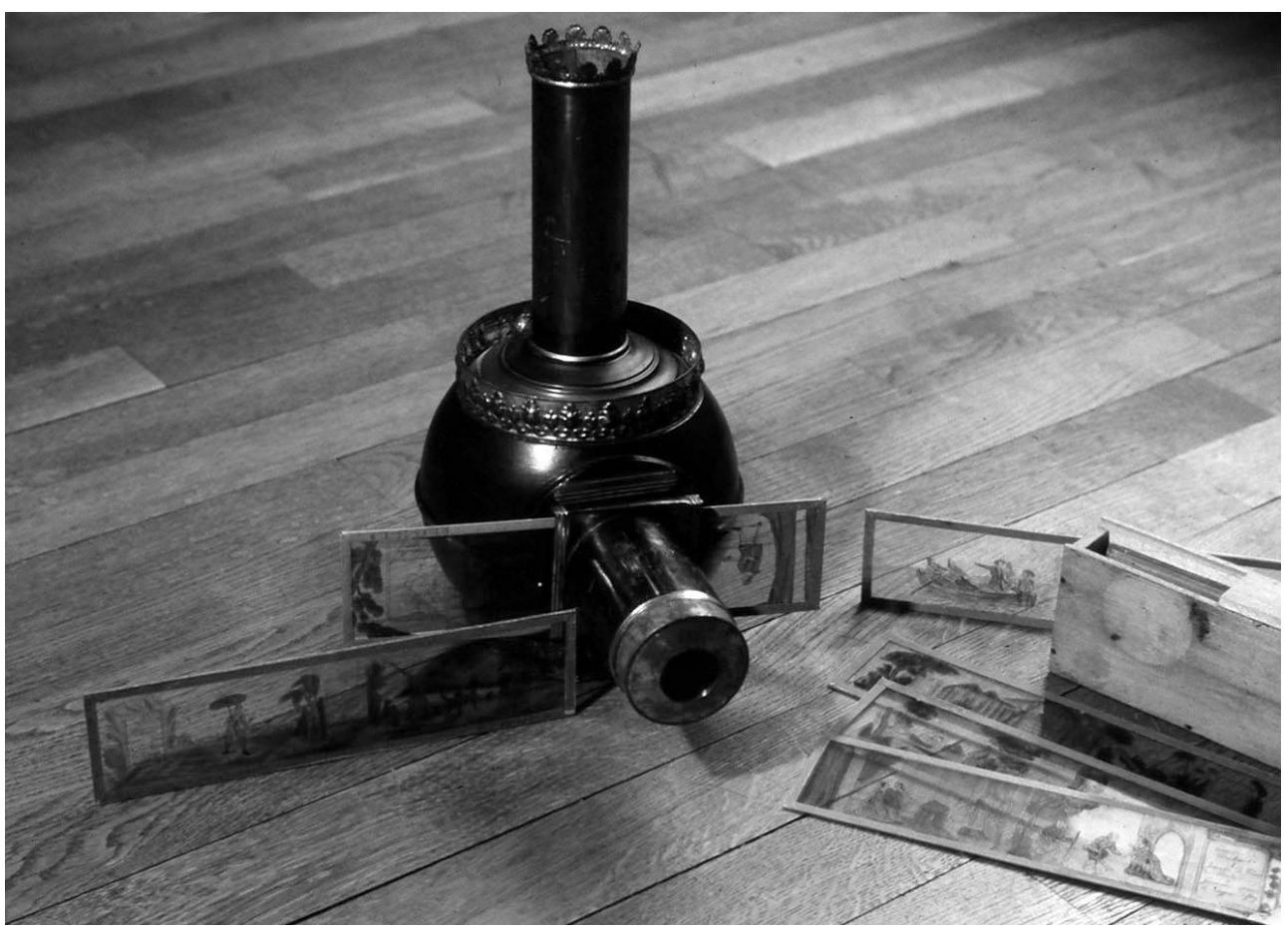

Installation, « Cinémas du futur », Lille, 2003.

\section{Le montreur colporteur}

Spectacle multimédia avant l'heure, la lanterne magique a engendré au début du $18^{\mathrm{e}}$ siècle une nouvelle figure de l'artiste, ou du moins un nouveau métier artistique - si déconsidéré soit-il - et aujourd'hui disparu: le lanterniste ambulant, projectionniste, chiffonnier-montreur, bonimenteur ou colporteur. Les dénominations sont nombreuses. C'est que lui reviennent différents rôles. Il peut être installé dans une foire, sur la place d'un village, accompagné parfois d'un singe ou d'une «marmotte en vie ", jouant d'une vielle ou d'un orgue de Barbarie. Il peut aussi déambuler dans les villes, coffret de la lanterne sur le dos ou transporté sur une charrette, pour projeter ses «tableaux magiques » à domicile. Ces extraits de boniment oratoire en décrivent le principe : «[...] Demandez la curiosité ! Faites monter chez vous la belle lanterne magique; il ne vous en coûtera pas plus de cinquante-cinq sols. [...] Attachez un drap blanc sur votre mur, et cependant appelez-moi par la fenêtre [...]. Moi, je viendrai avec mon appareil, et alors vous aurez du plaisir pour votre $\operatorname{argent}^{24} \ldots »$.

Ce type d'artiste saltimbanque n'a pas d'équivalent dans l'art actuel (à moins d'aller chercher du côté du théâtre de rue), mais sa polyvalence, son aspect homme-orchestre se retrouvent chez le performer ou l'artiste comme animateur, médiateur tel que le conçoivent Chris Hales et Teijo Pellinen dans leur spectacle de performance et vidéo interactive Cause and Effect ${ }^{25}$. Par ailleurs, le mode de monstration de la lanterne consistant à «faire monter l'art chez soi » reste très moderne : l'internet ne repose-t-il pas sur le même principe? Un regard rétrospectif nous permet de voir en la lanterne magique un art de la rue, mais aussi un art à domicile, mobile et accessible. 


\section{Le conférencier projectionniste} second temps, conférence-spectacle de vulgarisation scientifique; la lanterne n'est plus « magique » mais lanterne « de projection » instructive. À l'artiste montreur se substitue le conférencier. Petit à petit, il ne s'agit plus uniquement de dessins et de peintures : des vues photographiques sur verre sont projetées dès les années 1860 . Le photographe Eadweard Muybridge, lorsqu'il réalise ses premiers essais chronophotographiques aux États-Unis, se met à pratiquer en maître l'art de la conférence. Au moyen de la lanterne magique, il présente entre autres le résultat de ses expériences sur l'analyse du mouvement d'un cheval. D'après l'un de ses biographes, il passe « un très grand nombre d'images à intervalles rapprochés ${ }^{26} \%$. Puis il donne en 1880 la première conférenceprojection d'images animées grâce à un appareil de projection conçu à cet effet par luimême, ancêtre du projecteur cinématographique ${ }^{27}$. On pourrait parler de première séance de cinéma, si les vues projetées n'étaient pas des dessins réalisés d'après chronophotographies. Il demeure que ce type de spectacle est inédit pour l'époque, et connait un grand succès.

\section{L'artiste inventeur et installateur}

Lorsqu'elle n'est pas montrée sous forme de projection, la chronophotographie, à l'instar de la photographie du reste, n'est pas exposée dans un contexte artistique ${ }^{28}$. Elle - ou plutôt ses appareils de prise de vue et de projection - est visible dans des expositions et des Salons de type Exposition universelle. On n'y parle pas d'auteurs en tant qu'artistes, mais en tant qu'inventeurs ou savants. On retrouve aujourd'hui cette figure de l'artiste hybride dans des expositions telles qu'« Inventeurs! » (festival Exit 2005 à Créteil) qui mêle joyeux tintamarre populaire et recherches plus ou moins scientifiques.

Progressivement les vues chronophotographiques sont visionnées à l'aide de dispositifs issus des jouets et des machines d'optique. Leur mode de monstration se rapproche de l'installation telle qu'on la conçoit dans notre art contemporain : le kinétoscope d'Edison sert à visionner de manière individuelle (relativement voyeuriste) les premiers films cinématographiques, "films à trou de serrure ", destinés au public populaire des foires. Le spectateur regarde l'image à l'intérieur de la boîte, utilisée comme une machine à sous. Laurent Mannoni affirme que la notion de spectacle chronophotographique est établie avec le kinétoscope d'Edison en 1894-189529.

Appareil du même type, le phonoscope de Georges Demenÿ est une machine à visionner, mais aussi la première capable de projeter des vues chronophotographiques. Lors de l'Exposition photographique de 1892 - qui a lieu d'ailleurs au Palais des Beaux-Arts de Paris : un signe avant-coureur de la reconnaissance de la photographie comme un des beaux-arts -, Demenÿ présente son deuxième phonoscope. Il ne l'utilise pas comme projecteur, mais les conditions de monstration, très proches de celles de la projection, préfigurent en divers points nos black box actuelles ${ }^{30}$. Demenÿ est chargé par Marey d'installer le stand de la Station physiologique. Outre les appareils, statuettes, agrandissements de rigueur, il installe un phonoscope accompagné d'un dispositif sonore en fonctionnement permanent, ce qui est inédit. Il décrit le dispositif dans une lettre à Marey: «Je compte mettre une petite chambre noire avec mon zootrope par 
transparence caché et en mouvement. [...] J'ai réussi à construire quelques parleurs qui approchent de la perfection comme pureté. Les personnes qui voudront avoir l'illusion de la photographie parlante passeront dans un petite couloir d'un mètre de long et 80 centimètres de large fermé par un rideau et où l'on sera dans l'obscurité. Cela ne sera pas encombrant et aura un succès de nouveautés ${ }^{31} »$. Effectivement, comme le rapporte Laurent Mannoni, «la presse est dithyrambique. "Sous une tente dont les rideaux sont baissés, le spectateur applique son œil contre un verre et aperçoit une tête de jeune homme dont la bouche se desserre, s'ouvre, s'avance, se contracte, s'élargit, se ferme

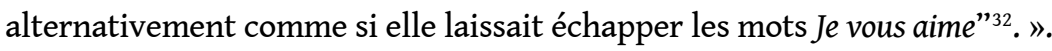

Le fonctionnement permanent de l'appareil - aujourd'hui on dirait que le film tourne en boucle, tandis qu'ici il est lui-même boucle, puisque les images sont disposées en couronne sur un disque -, la présence du son, l'occultation de l'appareil, l'installation d'un couloir, l'isolement spatial et phonique du spectateur immergé dans l'obscurité sont autant d'éléments qui présentent des similitudes avec nos installations vidéo et interactives.

\section{La station physiologique}

Un autre lieu d'exposition, voire le haut lieu d'exposition de la chronophotographie, est la Station physiologique ${ }^{33}$, dirigée par Marey et son assistant Georges Demenÿ. Elle n'est pas seulement un lieu de prises de vues et un laboratoire. Elle expose le résultat des recherches passées et en cours (sorte de work in progress) sous la forme d'appareils, de statuettes, de clichés photographiques, etc. "Un véritable cabinet des sciences et des curiosités, un musée cinématographique avant la lettre ", écrit Laurent Mannoni qui dépeint ainsi le chalet de la Station : «À la fin des années 1880, il sert presque de galerie d'exposition aux deux savants, qui montrent à leurs visiteurs le résultat de leurs recherches. Des physiologistes du monde entier rendent visite à la Station, mais aussi des peintres et des sculpteurs, pour copier les mouvements que les modèles ne peuvent poser. Les murs sont couverts de dessins, de graphiques, d'agrandissements photographiques, de tirages de plaques ou de films. Des panneaux montés en volets, retraçant l'évolution de la méthode graphique et de la chronophotographie, peuvent être feuilletés. Des systèmes étranges attirent l'attention des non-initiés : vélo relié à un appareil inscripteur, plateforme à air et à dynamomètre pour mesurer le saut de l'homme, machine à produire de la fumée, statuettes représentant la course de l'homme ou le vol de l'oiseau en plusieurs phases successives. Dans un coin, un énorme zootrope restitue, en tournant, l'illusion du mouvement d'un pigeon en plein $\operatorname{vol}^{34} \ldots$ ». La Station, même si cela n'est pas sa destination première, est un lieu d'exposition hybride, ouvert aux scientifiques et aux artistes, autorisés à feuilleter des images, et l'on imagine, à actionner entre autres le fameux zootrope.

\section{Le cabinet de curiosités}

Parmi les modes d'exposition caractéristiques du pré-cinéma, il faut compter le cabinet de curiosités, luxueux musée généralement privé et réservé à l'élite intellectuelle. Aux $18^{\mathrm{e}}$ et $19^{\mathrm{e}}$ siècles, sont en vogue dans les cabinets de curiosités toutes sortes d'inventions à la pointe de la technologie, liées aux arts et aux sciences, et principalement à la physique et à l'optique. On y retrouve entre autres la lanterne magique, qui se prête à des séances de 
projection privées, ainsi que des boîtes d'optique et de catoptrique, des prismes, anamorphoses, miroirs concaves et convexes, télescopes, etc. Le visiteur peut expérimenter et manipuler objets, images et jouets optiques en tous genres. Ce mode de monstration évoque inévitablement les expositions d'arts numériques et interactifs actuels, qui mêlent art et science, et dans lesquelles le spectateur est actif.

Par ailleurs, la collection est aujourd'hui une manière répandue d'appréhender le monde qui nous entoure. Privilégiée par la fonction de mémoire et la structure hypermédia du $c d, d u$ dvd-rom et de l'internet - il n'est qu'à recenser le nombre de musées virtuels existant à ce jour - la collection est même aux fondements de nombreuses démarches artitiques, comme l'attestent des œuvres interactives telles que Des souvenirs plein les poches de George Legrady, ou Mémoire de crayons de Jean-Louis Boissier.

Cette mise en regard entre pré-cinéma et art interactif sur le plan des modalités de monstration, des figures de l'artiste et des comportements du spectateur ne prétend pas à l'exhaustivité. D'autres dispositifs, d'autres formes de participation ou d'inclusion du spectateur à l'œuvre existent. À titre d'exemple, les panoramas des siècles derniers ${ }^{35}$ sont régulièrement cités comme référence des œuvres immersives actuelles. Par ailleurs, dans certains spectacles d'illusionnisme du $19^{\mathrm{e}}$ siècle, le spectateur était pris à témoin ou interpellé par le magicien pour se faire couper en morceaux, ou encore, téléporter (dématérialiser, numériser, pixelliser...).

L'accent a davantage été porté dans ce texte sur la relation à l'image dans les jouets, les machines optiques et les installations interactives.

Nous supposons qu'il existe une correspondance entre la disparition de l'intervention de la main dans l'acte de création des images technologiques au $19^{\mathrm{e}}$ siècle, et son apparition au même moment, dans la réception des images par le spectateur. De même, il semble qu'avec l'art interactif, la main soit passée de l'auteur au spectateur. En tant qu'art « technologique », l'art interactif partage divers points communs avec le pré-cinéma qui, en un sens, en constitue l'une des origines. Ainsi il serait souhaitable que le pré-cinéma, art "non noble", retrouve la place qui lui revient dans la préhistoire de l'image interactive, voire dans l'histoire de l'art. L'« art numérique " lui-même, "impur " par définition et tributaire de la science et des évolutions techniques, rencontre encore quelque résistance devant sa légitimation en tant qu'art. Ceci est dû sans doute au fait que le «numérique » se soit en quelque sorte autoproclamé « art » avant même de pénétrer le monde de l'art. De plus en plus, on le reconnaît comme une simple technologie plutôt que comme un art. Ce point de vue n'est pas forcément réducteur, puisque cela le positionne hors de toute catégorie, lui permettant d'appartenir plus aisément au monde de l'art contemporain. À cet égard, rappelons que la chronophotographie n'a pas eu besoin d'être officiellement considérée comme une pratique artistique pour entrer dans les livres d'histoire de l'art. À son époque, elle était avant tout une technologie d'avant-garde. 


\section{NOTES}

1. Il sera question ici de l'interactivité exogène, à savoir le dialogue homme-machine, et non de la relation endogène, interne aux machines.

2. Il s'agit de la version présentée lors la deuxième édition de " $1^{\text {er }}$ Contact ", festival d'art numérique à ciel ouvert d'Issy-les-Moulineaux en 2005.

3. Nous pensons à des pièces telles que Dompteurs de nuages (2005) de Florent Trochel - œuvre non dénuée de poésie pour autant, dans laquelle les déambulations du visiteur engendrent l'apparition de nuages sous ses pas.

4. Florence de Mèredieu, Arts et nouvelles technologies. Art vidéo, art numérique, Paris, Larousse, 2003, p. 97.

5. Présenté au festival d'art numérique à ciel ouvert, "1er Contact ", deuxième édition, Issy-lesMoulineaux, 2005.

6. Pour des précisions et des illustrations, voir Caroline Chik, « La fixité comme réalité de l'image animée ", dans Créer, jouer, échanger. Expériences de réseaux, actes de h2ptm'05, Paris, Hermès Lavoisier, 2005.

7. Métaphore chère à l'« accoucheur d'esprits numériques » Edmond Couchot, pour qui l'image de synthèse, faite d'immatérialité, rompt le cordon ombilical, le lien physique avec la réalité.

8. Jean-Louis Boissier, « Notes sur l'esthétique du virtuel ", dans La Relation comme forme. L'Interactivité en art, Genève, Mamco, 2004, p. 140.

9. Visible dans le cd-rom d'artistes Artintact n 1, Karlsruhe, zkm, 1994.

10. Propos recueillis par Martine Béguin et Jean-Paul Felley, dans Eric Lanz, Prix Brequet d'art contemporain, 1993, retranscrits sur le site internet du ciren à la page : http://www.ciren.org/ artifice/artifices_3/instal/Lanz.html.

11. Voir notamment Laurent Mannoni, Le Grand Art de la lumière et de l'ombre. Archéologie du cinéma, Paris, Nathan Université, 1994.

12. À titre indicatif la «Fée Électricité » fait sa première apprarition publique en 1881, à l'Exposition Internationale d'Électricité de Paris, et la France s'électrifie à partir des années 1920. Avant de supplanter la manivelle, elle cohabite avec elle lorsque les ampoules électriques se substituent aux chandelles des appareils nécessitant une source de lumière, tels que les projecteurs.

13. La périphrase est reprise du titre d'un ouvrage d'Athanase Kircher, Ars magna lucis et umbrae, Rome, Ludovigo Grignani, 1646.

14. Dominique Païni, préface de Laurent Mannoni, Trois siècles de cinéma. De la lanterne magique au Cinématographe, Paris, Cinémathèque Française, 1995, p. 10.

15. Voir Jeffrey Shaw, Peter Weibel (dir.), Future Cinema. The Cinematic Imaginary After Film, Karlsruhe, Cambridge, zkm / mit Press, 2003.

16. Propos recueillis dans Cinémas du futur. Édition 2004 (collectif), catalogue de l'exposition, Lille, Exhibitions International, 2003, p. 59.

17. http://www.zoebeloff.com.

18. Cette installation a été présentée au festival « Cinémas du futur », Lille, 2004.

19. Charles Baudelaire : «Le joujou est la première initiation de l'enfant à l'art, ou plutôt c'en est pour lui la première réalisation... ", dans « La morale du joujou », Le Monde littéraire, 17 avril 1853, retranscrit sur la page http://www.bmlisieux.com/litterature/baudelaire/moraljou.htm.

20. Jean-Louis Boissier, " Programmes interactifs ", La Relation comme forme. L'Interactivité en art, op. cit., p. 203. 
21. Les Allemands nomment le folioscope Daumenkino, (cinéma de pouce).

22. Boîte équipée d'une optique permettant de visionner ou de projeter des images peintes sur verre, fixes ou animées par superposition de plaques que l'on dit alors mécanisées.

23. Ségolène le Men, « Monsieur le Soleil et Madame la Lune ", dans Lanternes magiques. Tableaux transparents, Paris, rmn, 1995, p. 13.

24. Théodore de Banville, Petites Études - La Lanterne magique - Camées parisiens. La Comédie française. Avec un dessin de Georges Rochegrosse, Paris, G. Charpentier, 1883, p. 3 et 4, cité par Ségolène le Men, art. cit., p. 11.

25. Spectacle présenté à la Maison de la Villette lors du festival «Émergences » 2005.

26. Kevin Mac Donnell, Eadweard Muybridge, l'Homme qui a inventé l'image animée (trad. fr. P. Vieilhomme), Paris, Chêne, 1972, p. 25.

27. Muybridge a donné différents noms à son projecteur : zoographiscope, zoogyroscope, zoopraxinoscope puis zoopraxiscope.

28. La première galerie de photographie considérée comme un art moderne n'ouvrira qu'en 1905 sous l'égide d'Alfred Stieglitz. Avant cette date, les photographies sont exposées dans des Salons et dans quelques rares expositions de musées (Paris, Turin, Glasgow, Düsseldorf et Chicago).

29. Laurent Mannoni, Le Grand Art de la lumière et de l'ombre. Archéologie du cinéma, op. cit., p. 372.

30. La black box ou le black cube désignent l'espace clos de projection des images animées et interactives nécessitant l'obscurité du lieu, par opposition au white cube.

31. Laurent Mannoni, Georges Demenÿ. Pionnier du cinéma, Douai, Pagine, 1997, p. 49.

32. ibid., p. 50.

33. Ce lieu a été détruit depuis.

34. ibid., p. 25-26.

35. Les panoramas des $18^{\mathrm{e}}$ et $19^{\mathrm{e}}$ siècles désignent de vastes tableaux circulaires peints en trompe-l'œil, englobant le spectateur situé au centre.

\section{RÉSUMÉS}

Dans l'art interactif, de nouveaux rapports physiques se créent entre l'œuvre et le spectateur jusqu'à y inclure ce dernier. D'autres dispositifs reposent sur la notion de contact, faisant de la main une interface et de l'œuvre un jouet manipulable. Un rapprochement entre pratiques interactives et jouets optiques pré-cinématographiques laisse apparaître des similitudes dans certaines figures hybrides de l'artiste et dans les modes de monstration de deux pratiques technologiques pourtant éloignées de plusieurs siècles.

In interactive art, new physical configurations are created between the artwork and the spectator. Some participation modes tend to reduce the spatial distance between these two, even including the spectator in the artwork itself. Other situations rely on the notion of contact ; using the hand as an interface and the artwork as a toy that can be played with. A parallel between interactive artworks and pre-cinema optical toys shows similarities in some hybrid figures of the artist and in two technological practices, centuries apart. 


\section{AUTEUR}

\section{CAROLINE CHIK}

Doctorante en Arts Plastiques, Sciences et Technologies des Arts à Paris 8, artiste multimédia 